\title{
Cumulative Effects of Psychologic Distress, Visceral Hypersensitivity, and Abnormal Transit on Patient-reported Outcomes in Irritable Bowel Syndrome
}

\author{
Magnus Simrén, ${ }^{1,2}$ Hans Törnblom, ${ }^{1}$ Olafur S. Palsson, ${ }^{2}$ Lukas Van Oudenhove, ${ }^{3}$ \\ William E. Whitehead, ${ }^{2}$ and Jan Tack $^{3}$ \\ ${ }^{1}$ University of Gothenburg, Gothenburg, Sweden; ${ }^{2}$ University of North Carolina at Chapel Hill, Chapel Hill, North Carolina; and \\ ${ }^{3}$ Katholieke Universiteit Leuven, Leuven, Belgium
}

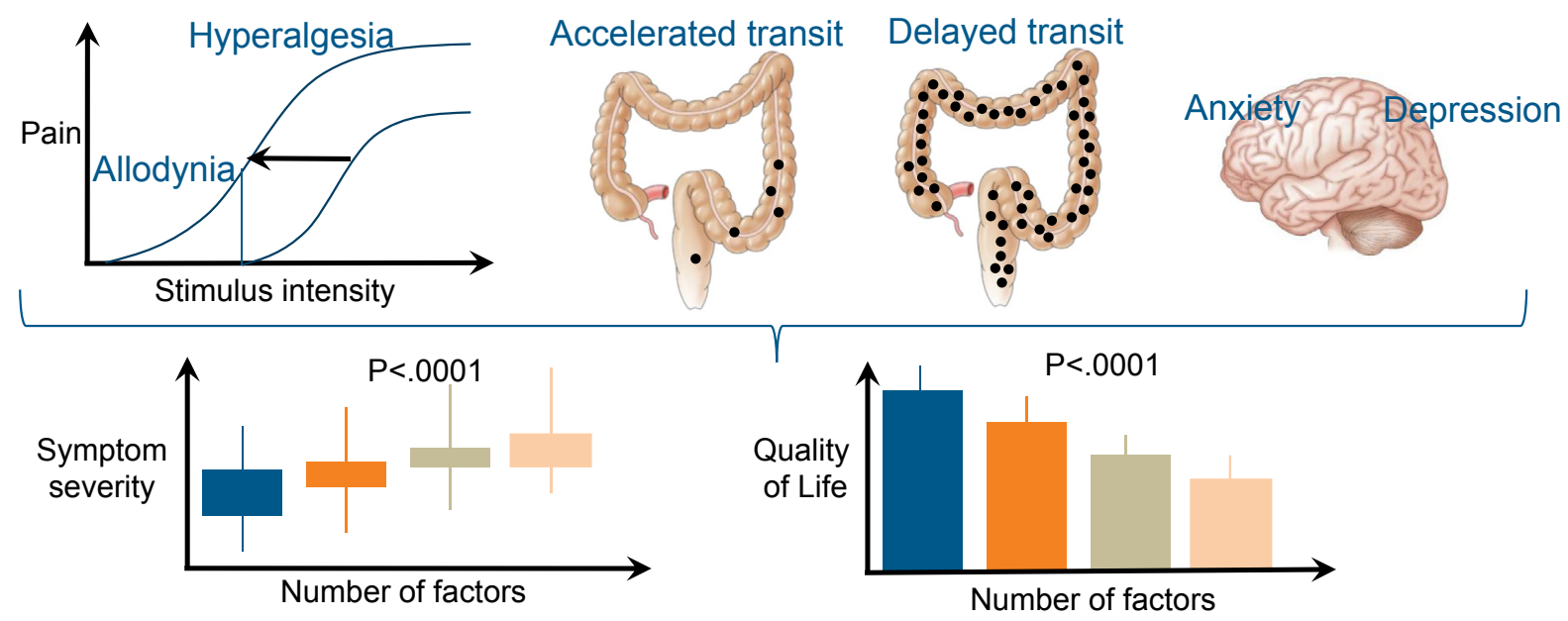

BACKGROUND \& AIMS: Little is known about the link between pathophysiologic factors and symptoms of irritable bowel syndrome (IBS), or whether these factors have cumulative effects on patient-reported outcomes (PROs). We investigated whether pathophysiologic alterations associated with IBS have cumulative or independent effects on PROs. METHODS: We performed a retrospective analysis of data from 3 cohorts of patients with IBS ( $\mathrm{n}=407 ; 74 \%$ female; mean age, $36 \pm 12$ years), based on Rome II or Rome III criteria, seen at a specialized unit for functional gastrointestinal disorders in Sweden from 2002 through 2014. All patients underwent assessments of colonic transit time (radiopaque markers); compliance, allodynia, and hyperalgesia (rectal barostat); anxiety and depression (Hospital Anxiety and Depression scale), as pathophysiologic factors. Dysfunction was defined by available normal values. PROs included IBS symptom severity, somatic symptom severity, and disease-specific quality of life. RESULTS: Allodynia was observed in $36 \%$ of patients, hyperalgesia in 22\%, accelerated colonic transit in $18 \%$, delayed transit in 7\%, anxiety in 52\%, and depression in $24 \%$ : each of these factors was associated with severity of at least 1 symptom of IBS. Rectal compliance was not associated with more severe symptoms of IBS. At least 3 pathophysiologic factors were present in $20 \%$ of patients, 2 in $30 \%, 1$ in $31 \%$, and none in $18 \%$. With increasing number of pathophysiologic abnormalities, there was a gradual increase in IBS symptom severity $(P<.0001)$ and somatic symptom severity $(P<$ $.0001)$, and a gradual reduction in quality of life $(P<.0001)$. CONCLUSION: Visceral hypersensitivity, including allodynia and hyperalgesia, abnormal colonic transit, and psychologic factors are all associated with IBS symptoms. These factors have a cumulative effect on gastrointestinal and nongastrointestinal symptoms, as well as on quality of life, in patients with IBS and are therefore relevant treatment targets.

Keywords: Visceral Hypersensitivity; Colonic Transit; Psychologic Distress; Patient-Reported Outcomes.

Abbreviations used in this paper: ANOVA, analysis of variance; GI, gastrointestinal; GSRS-IBS, Gastrointestinal Symptom Rating Scale-IBS; HAD, Hospital Anxiety and Depression scale; IBS, irritable bowel syndrome; IBSQOL, Irritable Bowel Syndrome Quality of Life Questionnaire; IBS-SSS, IBS severity scoring system; PHQ-15, Patient Health Questionnaire-15; PRO, patient-reported outcome; VAS, visual analogue scale. 


\section{WHAT YOU NEED TO KNOW}

\section{BACKGROUND AND CONTEXT}

Little is known about the link between pathophysiologic factors and symptoms of irritable bowel syndrome (IBS), or whether these factors have cumulative effects on patient-reported outcomes.

\section{NEW FINDINGS}

Visceral hypersensitivity, including allodynia and hyperalgesia, abnormal colonic transit, and psychologic factors are all associated with IBS symptoms, and have a cumulative effect on symptoms and QOL in IBS.

\section{LIMITATIONS}

Some important pathophysiologic factors in IBS, eg, factors in the gut microenvironment, were not investigated in this study.

IMPACT

The cumulative effect of visceral hypersensitivity, abnormal colonic transit, and psychologic factors on patient-reported outcomes in IBS highlight the importance of gut-brain interactions, and indicate that these pathophysiologic factors constitute relevant treatment targets.

$\mathrm{I}$ rritable bowel syndrome (IBS) is a common functional gastrointestinal (GI) disorder, characterized by abdominal pain associated with abnormal bowel habit, but patients also often suffer from other GI and non-GI symptoms, including psychologic symptoms and psychiatric comorbidity. ${ }^{1}$ The efficacy of available therapeutic options for this large group of patients is limited, which partly relates to the fact that mechanisms underlying symptom generation are incompletely known. ${ }^{2,3}$ The pathophysiology of IBS is considered to be complex and most likely multifactorial, and abnormal gut-brain interactions are considered to be of great importance. ${ }^{4}$ of the individual pathophysiologic factors, visceral hypersensitivity, ${ }^{5-8}$ abnormal GI motor function, ${ }^{5,6,9}$ and abnormal brain function with abnormal central processing of visceral afferent signals, which may be driven by psychologic symptoms modulating the processing and perception of these signals, ${ }^{10-12}$ have all been widely studied and are believed to be of importance for symptoms in subsets of patients with IBS. Factors in the gut microenvironment are increasingly being recognized as belonging in the pathophysiologic model of IBS as well. ${ }^{4,13-17}$ However, the relevance of these factors for symptom generation and severity in IBS is largely unknown.

Visceral hypersensitivity is one of the best characterized pathophysiologic abnormalities in IBS. It consists of 2 components: allodynia, which means that an innocuous stimulus is perceived as painful, and hyperalgesia, which means an enhanced pain response (ie, a painful stimulus is felt more intensely). ${ }^{18}$ In IBS, most studies have focused on a lowered pain threshold as a marker of visceral hypersensitivity, which reflects allodynia. ${ }^{6-8,19,20}$ However, increased perceived intensity of a painful stimulus (ie, hyperalgesia) also has been reported. ${ }^{5,7}$ Several studies have demonstrated an association between presence of visceral hypersensitivity, mostly defined as lowered rectal or colonic pain thresholds, and IBS symptom severity, ${ }^{6,7,21}$ and this can be observed even after controlling for psychologic symptoms and general symptom reporting. ${ }^{22}$ There are also studies that have demonstrated an association between intensity ratings (visual analogue scales, VAS) during painful rectal balloon distensions, reflecting hyperalgesia, and IBS symptom severity. ${ }^{7}$ Moreover, in studies assessing colorectal sensitivity, in which compliance is frequently measured as well, reduced rectal compliance has been reported in patients with IBS in some studies, but the association of this with IBS symptom severity is also less well established. $^{20,21}$

Abnormal GI motor function is another hallmark pathophysiologic factor in IBS, although a specific motor pattern for IBS has not been identified and clear correlations between motility findings and symptoms have been difficult to demonstrate. $^{23,24}$ However, measurement of colonic or oroanal transit time as an indirect measure of colonic motility shows more consistent findings, with colonic transit abnormalities being associated with IBS subtype in that accelerated transit is seen predominantly in patients with diarrhea, and delayed transit in patients with constipation. ${ }^{5,9,25-27}$ Moreover, even though it has been hard to associate specific motility abnormalities in IBS with pain severity, ${ }^{5}$ some studies have found transit to be weakly but significantly associated with pain and/or bloating, in addition to the association with bowel habit abnormalities. ${ }^{9,27}$

Psychologic symptoms, such as anxiety and depressive symptoms, in more severe cases fulfilling the criteria for comorbid anxiety or depressive disorders, are common in patients with IBS, ${ }^{28}$ and the presence of comorbid anxiety and depressive symptoms or syndromes is associated with the overall GI symptomatology. ${ }^{29}$ In the latest edition of the diagnostic criteria for functional GI disorders, the Rome IV criteria, these disorders are referred to as disorders of gutbrain interaction, highlighting the importance of not only the gut but also the brain in symptom generation in these conditions. ${ }^{30}$ Indeed, altered central nervous system or brain function has been demonstrated in patients with IBS compared with control subjects using brain imaging. ${ }^{11}$ However, in one study the group brain differences between IBS and healthy controls disappeared when controlling for anxiety and depression, ${ }^{31}$ which indirectly suggests that measures of anxiety and depression can be used as proxy measures for abnormal brain function in patients with IBS, although abnormal brain dysfunction in IBS may indeed exist irrespective of anxiety and depression according to other studies. ${ }^{32}$

Few studies have investigated how the different pathophysiologic factors in IBS relate to each other and how they interact to influence symptoms, that is, whether they are individually independent pathophysiologic factors or whether they are correlated in their presence and impact on symptoms. Visceral hypersensitivity is associated with psychologic factors, but its association with GI symptoms is not solely explained by psychologic factors. ${ }^{7,22,33}$ The association between visceral hypersensitivity and GI motility has 
been assessed in a small number of trials with inconsistent results and with no attempts to assess their combined influence on symptoms. ${ }^{6,20}$ The association between GI motility and psychologic factors such as stress, anxiety and depression has been established with different methodologies, ${ }^{34,35}$ but their combined influence on symptoms has not been evaluated. In line with the current view on functional GI disorders as disorders of gut-brain interaction, ${ }^{30}$ it seems reasonable to hypothesize that different factors involved in the complex pathophysiology of IBS may interact and influence symptoms in a cumulative fashion in these patients, but evidence from studies supporting this is not available. However, it also may be possible that these factors do not act cumulatively but instead influence symptoms in an independent way, which was suggested for colorectal motility and visceral hypersensitivity in one study. ${ }^{6}$

Therefore, the aim of the present study was to evaluate whether key pathophysiologic factors have a cumulative effect on symptoms and disease-specific quality of life in IBS. Specifically we aimed to determine the presence of visceral hypersensitivity as reflected by allodynia and hyperalgesia, abnormal rectal compliance, delayed or accelerated colonic transit, reflecting abnormal colonic motility, and anxiety and depression symptoms, reflecting abnormal brain function, and to evaluate how the presence of one or more of these influence relevant patient-reported outcomes (PROs) in IBS, that is, IBS and overall somatic symptom severity, and IBS-specific quality of life.

\section{Materials and methods}

\section{Subjects}

We included subjects with IBS aged 18 to 70 years from 3 prospective cohort studies that were conducted to assess the relevance of different pathophysiologic factors for IBS symptoms. For this study, we included only subjects who had completed measurements of rectal sensitivity and compliance, colonic (eg, oroanal) transit time, anxiety and depression, severity of GI and overall somatic symptoms and diseasespecific quality of life (see later in this article for details; investigations and questionnaires completed within a 2-week period). The patients in the first cohort were included between 2002 and 2007, patients in the second cohort between 2007 and 2010, and patients in the third cohort between 2010 and 2014. The patients were included at a specialized unit for functional GI disorders at the University of Gothenburg, Sweden, and predominantly referred to the unit from primary care, but self-referral was also accepted. All patients were seen by a gastroenterologist (HT or MS) who confirmed the diagnosis based on a typical clinical presentation and additional tests if needed. However, most patients had already undergone sufficient examinations before referral. All patients fulfilled the current diagnostic criteria at the time of inclusion, that is, the Rome II criteria up to $2006,{ }^{36}$ and the Rome III criteria thereafter. ${ }^{37}$ The patients were subtyped according to predominant bowel habit based on the Rome II or III recommendations, ${ }^{36,37}$ and defined as diarrhea-predominant or constipationpredominant IBS, whereas patients with IBS with mixed/ alternating type and unsubtyped IBS were combined into 1 group for the analyses in this study (ie, nonconstipation- or diarrhea-predominant IBS; nonC or nonD). Exclusion criteria were as follows: other GI disease(s) explaining the patient's symptoms; other severe disease(s) such as malignancy, severe heart disease, kidney disease, or neurological disease; symptoms indicating other severe disease(s) such as GI bleeding, weight loss not explained by altered eating habits; fever; severe psychiatric disease being the dominant clinical problem; a history of drug or alcohol abuse within 6 months before enrollment; or pregnancy at the time of the study. All subjects refrained from the use of drugs affecting GI function at least 48 hours before the investigations. The studies were approved by the Regional Ethical Review Board in Gothenburg, and all patients gave verbal and written informed consent before any study-related procedures were undertaken.

\section{Pathophysiologic Measures}

In all studies, a rectal barostat test, a measure of colonic transit (eg, oroanal transit time), and a questionnaire to assess anxiety and depressive symptom severity were included. However, the protocols used differed slightly between study cohorts (see later in this article). Other measures of potential relevance for IBS pathophysiology were included in the study protocols as well, but these were not consistent between the study protocols, have been reported elsewhere, ${ }^{16,17,38-45}$ and were not included in the analyses presented here. A proportion of the subjects have been included in previous publications focusing on the individual abnormalities assessed in this study, ${ }^{9,20,22}$ but not including the specific analyses performed in this study.

Oroanal transit time. The subjects ingested 10 radiopaque markers every morning for 5 days. On the sixth day they ingested 5 radiopaque markers in the morning and 5 at 8 PM. On the seventh day, the subject came to the laboratory at 8 $\mathrm{Am}$ and the remaining radiopaque markers were counted using fluoroscopy (Exposcop 7000 Compact; Ziehm GmbH, Nüremberg, Germany). Oroanal transit time (days) was calculated by dividing the number of remaining radiopaque markers by 10 (ie, the daily dose of radiopaque markers). To define accelerated and delayed oroanal transit time we used normal values (5th and 95th percentile) obtained from investigations in 199 healthy volunteers at our laboratory (women 0.9-3.9 days; men 0.7-2.1 days)..$^{9,27,46-48}$

Rectal barostat. Two different rectal barostat protocols were used to study rectal sensitivity and compliance. In the first and second cohorts we used a protocol with phasic isobaric distensions lasting 30 seconds followed by a 30 -second interval at the individual operating pressure. ${ }^{7,20}$ Distensions were performed with stepwise increments starting at the individual operating pressure and increasing $5 \mathrm{~mm} \mathrm{Hg}$ until the subject reported pain or when a pressure of $70 \mathrm{~mm} \mathrm{Hg}$ was reached. At the end of this protocol (ie, at the pain threshold), the intensity of pain was rated by the patients using VAS. For this study, the pain threshold ( $\mathrm{mm} \mathrm{Hg}$ ) was used as a measure of allodynia, and the VAS pain intensity rating at the pain threshold was used as a measure of hyperalgesia. The first 5 distension steps of the pressure-volume curves were used for analysis of compliance, as the initial part of the curve is linear, which enables quantification of dynamic compliance as the linear slope of the curve. Furthermore, data up to the fifth distension step were available in most subjects, because most subjects had not yet reached the pain threshold at this step. ${ }^{20}$ 
To identify patients with allodynia, hyperalgesia, and increased/decreased compliance, respectively, we used normal values (5th and 95th percentile) obtained from investigations in 35 healthy volunteers at our laboratory (pain threshold $<31$ $\mathrm{mm} \mathrm{Hg}$; pain intensity at pain threshold $>54 \mathrm{~mm}$; compliance 2.9-10.4 $\mathrm{mL} / \mathrm{mm} \mathrm{Hg})^{7,20,22}$ In the third cohort, allodynia (pain threshold) and compliance were measured by using ramp inflations starting from $0 \mathrm{~mm} \mathrm{Hg}$ with $4 \mathrm{~mm} \mathrm{Hg}$ increments every minute until the subject reported pain. ${ }^{22,49}$ The balloon pressure at half the maximum observed volume was used as a summary value for compliance, whereby a smaller pressure corresponds to higher compliance. ${ }^{49}$ After the ramp inflations, 60 -second phasic distensions of $12,24,36$, and $48 \mathrm{~mm} \mathrm{Hg}$ above the operating pressure were each applied once in random order. The subjects rated the intensity of sensations of gas, urgency, discomfort, and pain at each of these distensions. For the purpose of this study, the pain threshold (mm Hg) was used as a measure of allodynia, and pain ratings at the $24 \mathrm{~mm}$ $\mathrm{Hg}$ distension were used as measure of hyperalgesia, because this was in the painful range for most subjects. To identify patients with allodynia, hyperalgesia, and increased/decreased compliance, respectively, we used normal values (5th and 95th percentile) obtained from investigations in 50 healthy volunteers at our laboratory (pain threshold $<20 \mathrm{~mm} \mathrm{Hg}$; pain intensity at $24 \mathrm{~mm} \mathrm{Hg}$ phasic distension $>76 \mathrm{~mm}$; compliance (pressure at half maximal volume): $12-27 \mathrm{~mm} \mathrm{Hg}$ ). ${ }^{22}$

Anxiety and depression. The presence of anxiety and/ or depressive symptoms was used as a proxy measure for abnormal central nervous system or brain function, hence abnormal central processing of visceral afferent signals, in this study. All patients completed the widely used and thoroughly validated Hospital Anxiety and Depression scale (HAD), and the validated cutoff score of $>7$ on either the anxiety or depression subscales was used to define abnormality. ${ }^{50,51}$

\section{PRO Instruments}

In all 3 cohorts, the patients completed questionnaires to assess IBS symptom severity, overall somatic symptom severity, and disease-specific quality of life. However, the questionnaires used differed between the cohorts (see later in this article).

IBS symptoms. In the first cohort, the Gastrointestinal Symptom Rating Scale-IBS (GSRS-IBS) was used to determine IBS symptom severity. GSRS-IBS consists of 13 questions, summarized in 5 domains (abdominal pain, bloating, constipation, diarrhea, and satiety). ${ }^{52}$ In the second and the third cohort, IBS symptom severity was determined using the IBS severity scoring system (IBS-SSS), and this questionnaire is based on 5 items: severity and frequency of abdominal pain, severity of abdominal distension, dissatisfaction with bowel habits, and interference of IBS with daily life. ${ }^{53}$ The patients also completed a stool diary for 1 week using the Bristol Stool Form scale ${ }^{54}$; from these diaries, information about average stool frequency and average stool consistency over a week was obtained.

Overall somatic symptoms. We used 2 different measures of overall somatic symptom severity, often referred to as somatization. Patients in the first cohort completed the Symptom Checklist-90, a questionnaire developed to measure psychological symptom patterns of psychiatric and medical patients. ${ }^{55}$ It consists of 9 primary symptom dimensions, but for this study we used only the somatization subscale. In the second and third cohorts, the Patient Health Questionnaire-15
(PHQ-15) was used. PHQ-15 assesses the perceived severity of 15 different somatic symptoms, which are summarized into an overall somatic symptom severity score. ${ }^{56}$

Disease-specific quality of life. IBS-specific quality of life was measured in all 3 patient cohorts with the Irritable Bowel Syndrome Quality of Life Questionnaire (IBSQOL). ${ }^{57}$ This 30 -item questionnaire measures 9 quality of life domains found to be of relevance for IBS: emotional health, mental health, sleep, energy, physical functioning, food/diet, social functioning, physical role, and sexual relations. Each scale score is transformed to a scale of 0 to 100 , with 100 representing the best possible quality of life.

\section{Data Analysis and Statistics}

The first step in the data analysis was to identify patients with IBS with pathophysiologic abnormalities. Patients with results from the barostat and transit tests used in this study that were outside the 5th and/or 95th percentile in healthy control subjects, as appropriate, were defined as having rectal allodynia, rectal hyperalgesia, increased/decreased rectal compliance, or accelerated/delayed transit, respectively, and validated cutoff values for the HAD questionnaire were used to identify patients with anxiety and depression (see previously). ${ }^{50,51}$ To be included in the next step of the analyses, a pathophysiologic factor had to be associated with the severity of $\geq 1$ IBS-related symptom (IBS-SSS pain frequency and severity, bloating severity, dissatisfaction with bowel habits; GSRS-IBS abdominal pain, bloating, constipation, diarrhea; average stool frequency and consistency from Bristol Stool Form stool diaries), and in the expected direction, that is, more severe symptoms in patients with the pathophysiologic factors than in those without. This was tested with independent samples $t$ tests between subjects with and without the pathophysiologic abnormality. For the identification of relevant pathophysiologic factors to be included in the next step in the analyses, we used uncorrected $P$ values $<.05$ as the cutoff, but we also performed false discovery rate correction for multiple testing to indicate $P$ values that survived false discovery rate correction. The number of pathophysiologic abnormalities in each patient was thereafter defined. To test the hypothesis that the pathophysiologic abnormalities have a cumulative effect on PROs, 1-way between-groups analysis of variance (ANOVA) with linear trend analyses to analyze the presence of a linear trend were performed, ${ }^{58}$ with number of pathophysiologic abnormalities as independent variable and the patient-reported outcome variable (IBS symptom severity, overall somatic symptom severity or IBS-specific quality of life) as the dependent variable. Effect size was evaluated using the following definition: small: partial eta squared 0.01; medium: partial eta squared 0.06; large: partial eta squared $0.14 .^{59}$ To be able to use single measures for IBS and overall somatic symptom severity for these analyses across all 3 patient cohorts, scores from the questionnaires measuring these variables in the studies (IBS-SSS and GSRS-IBS; Symptom Checklist-90 somatization subscale, and PHQ-15) $)^{52,53,55,56}$ were transformed to $Z$ scores (defining how many standard deviations a value is from the mean, which is set to 0 ), and those $z$ scores were thereafter used as the IBS and somatic symptom severity variables for all patients. In addition to analyses performed in the entire cohort, we repeated the linear trend analyses in the 3 individual IBS cohorts to assess congruence of findings across the cohorts. 
Table 1.Demographic and Disease-related Information

\begin{tabular}{|c|c|c|c|c|c|}
\hline & $\begin{array}{l}\text { Whole IBS cohort } \\
\qquad(\mathrm{n}=407)\end{array}$ & $\begin{array}{l}\text { IBS cohort } 1 \\
(n=137)\end{array}$ & $\begin{array}{l}\text { IBS cohort } 2 \\
(n=128)\end{array}$ & $\begin{array}{l}\text { IBS cohort } 3 \\
(n=142)\end{array}$ & $P^{a}$ \\
\hline Age, $y r$, mean $\pm \mathrm{SD}$ (range) & $36.0 \pm 12.0(19-67)$ & $36.8 \pm 12.2(19-67)$ & $36.8 \pm 12.2(19-67)$ & $34.4 \pm 11.5(19-67)$ & .17 \\
\hline IBS subtype (C/D/nonCnonD) & $99 / 145 / 163$ & $25 / 47 / 65$ & $35 / 41 / 49$ & $39 / 54 / 49$ & .18 \\
\hline HAD anxiety & $8.1 \pm 4.6$ & $7.9 \pm 4.7$ & $8.1 \pm 4.5$ & $8.3 \pm 4.5$ & .81 \\
\hline HAD depression & $5.2 \pm 3.7$ & $5.1 \pm 3.6$ & $5.3 \pm 3.9$ & $5.1 \pm 3.5$ & .86 \\
\hline Oroanal transit time, $d$ & $1.6 \pm 1.0$ & $1.7 \pm 1.1$ & $1.7 \pm 1.0$ & $1.5 \pm 1.1$ & .38 \\
\hline Rectal pain threshold, $\mathrm{mm} \mathrm{Hg}$ (protocol 1) & NA & $34 \pm 13$ & $31 \pm 13$ & NA & .02 \\
\hline $\begin{array}{l}\text { Rectal pain intensity, } \mathrm{mm} \\
\text { (at pain threshold; protocol 1) }\end{array}$ & NA & $33 \pm 23$ & $34 \pm 25$ & NA & 69 \\
\hline Rectal pain threshold, $\mathrm{mm} \mathrm{Hg}$ (protocol 2) & NA & NA & NA & $27 \pm 8$ & NA \\
\hline Rectal pain intensity, $\mathrm{mm}$ & NA & NA & NA & $48 \pm 33$ & NA \\
\hline
\end{tabular}

(at $24 \mathrm{~mm} \mathrm{Hg}$; protocol 1)

IBS-C, constipation-predominant IBS; IBS-D, diarrhea-predominant IBS; IBSnonCnonD, nonconstipation or diarrheapredominant IBS, hence patients with IBS mixed/alternating type and unsubtyped IBS; SD, standard deviation.

${ }^{a}$ ANOVA or $\chi^{2}$ test among the 3 different cohorts.

We also used an alternative grouping of pathophysiologic abnormalities, whereby we identified patients with psychologic distress (anxiety and/or depression using the same cutoff values as described previously), hypersensitivity (allodynia and/or hyperalgesia, defined as described previously) and abnormal transit (delayed or accelerated, as described previously). The same analytical approach as described previously regarding association between the number of these
Figure 1. (A) Proportion of subjects with pathophysiologic abnormalities relevant for IBS symptoms based on normal values in healthy volunteers (allodynia, hyperalgesia, transit abnormalities) and validated questionnaire cutoff scores (anxiety and depression), and $(B)$ proportion of subjects with different numbers of pathophysiologic abnormalities relevant for IBS symptoms: allodynia, hyperalgesia, delayed/ accelerated oroanal transit, anxiety, and depression.

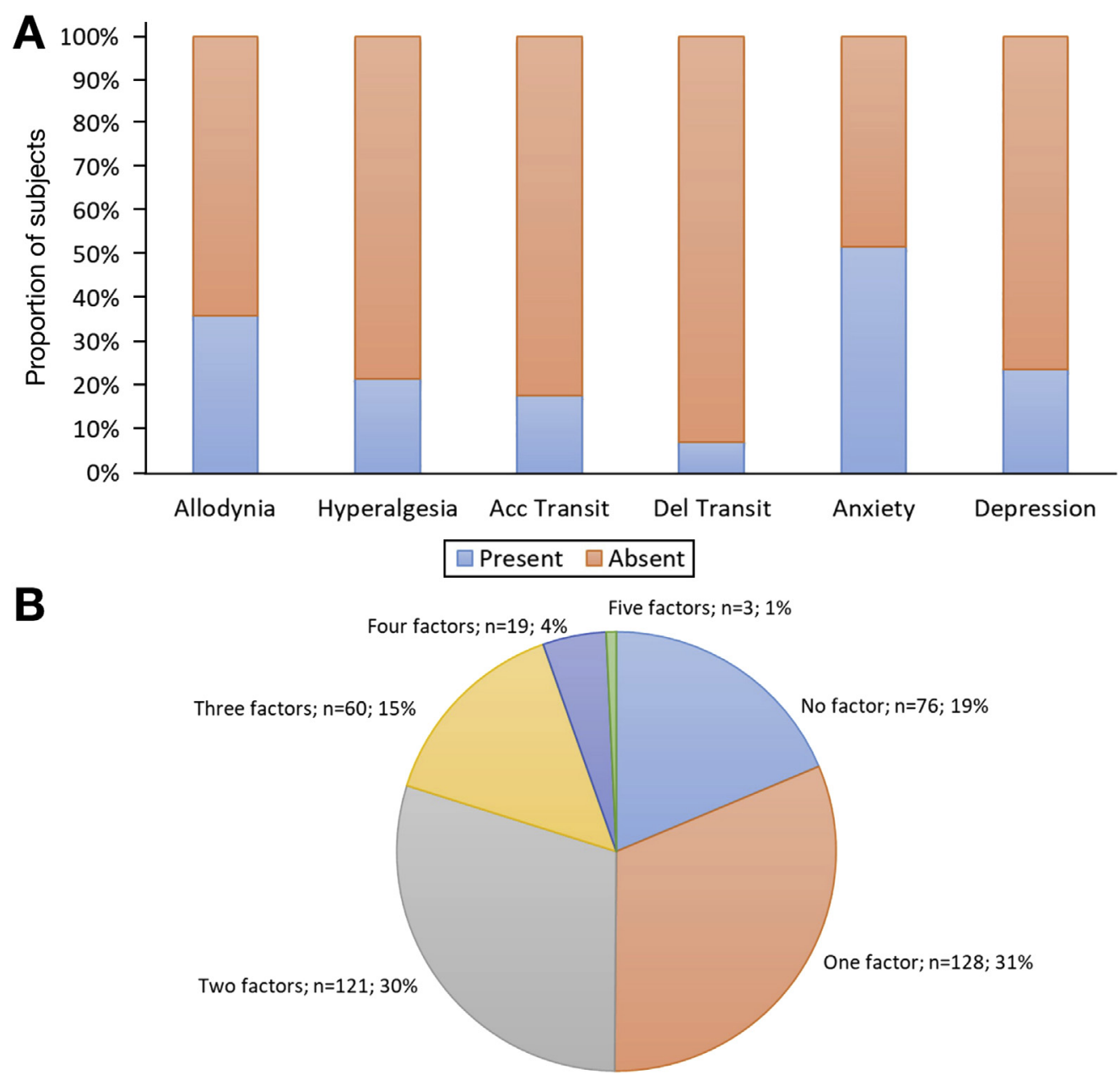


pathophysiologic factors and patient-reported outcome variables were also used for these factors. In addition, we performed multivariate linear regression models with presence of abnormal transit, hypersensitivity, and psychologic distress as dichotomous independent variables in 2 models, one with IBS symptom severity and another with overall somatic symptom severity as the dependent variable. In general a $P$-value $<.05$ was considered statistically significant, and data are presented as mean \pm standard deviation and proportions as appropriate.

\section{Results}

\section{Subjects}

We included 407 subjects with IBS; 137 in cohort 1 diagnosed according to the Rome II criteria, and 128 and 142 in cohorts 2 and 3, respectively, both diagnosed according to the Rome III criteria. Demographic and diseaserelated information can be seen in Table 1 . There were no differences in mean age, gender distribution, or distribution of IBS subtypes among the 3 cohorts. Moreover, except for a slightly lower rectal pain threshold in cohort 2 than in cohort 1, all the parameters used in the main analyses in this study were equivalent across the cohorts, that is, oroanal transit time, overall IBS symptom severity $z$ scores, somatic symptom severity $Z$ scores, and anxiety and depression scores (HAD). The rectal pain threshold in cohort 3 was numerically lower, and the rectal pain intensity numerically higher than in cohorts 1 and 2, but this was expected, because a different barostat protocol with different normal values was used in this cohort. Therefore, the 3 cohorts are combined in all analyses in this report. Oroanal transit time differed between IBS subtypes (ANOVA, $P<.0001$; post hoc comparisons, Bonferroni adjusted, $P<.05$ between all groups), and patients with IBSnonCnonD had lower HAD depression scores (ANOVA, $P=.02$; post hoc comparisons, Bonferroni adjusted, $P<.05$ between IBSnonCnonD and constipation-predominant IBS only), but otherwise the pathophysiologic measures and PROs were similar among IBS subtypes (Supplementary Table 1)

\section{Pathophysiologic Factors}

Based on normal values and validated cutoff scores, allodynia was found in 145 patients (36\%), hyperalgesia in 86 patients $(22 \%)$, accelerated oroanal transit in 73 patients (18\%), delayed oroanal transit in 29 patients (7\%), anxiety in 211 patients (52\%), and depression in 97 patients $(24 \%)$ (Figure $1 A$ ). All of these factors were associated with the severity of at least 1 IBS symptom, that is, more severe symptoms in patients with vs without the pathophysiologic factor (Table 2). Anxiety and depression were associated with the largest number of IBS symptoms, transit abnormalities primarily with bowel habit disturbances, and allodynia and hyperalgesia with pain and bowel habits. Rectal compliance, which was increased in 58 patients (14\%) and reduced in 38 patients (9\%) was not associated with more severe IBS symptoms, and therefore not included in the subsequent analyses. At least 3 pathophysiologic abnormalities relevant for symptoms were present in 82 patients (20.1\%), 2 abnormalities in 121 patients (29.7\%), 1 abnormality in 28 patients (31.4\%), and 76 patients $(18.7 \%)$ had none of the abnormalities under consideration (Figure $1 B$ ). There were no associations between the number of pathophysiologic abnormalities and sex $(P=.12)$, age $(P=.15)$, or IBS subtype $(P=.21)$.

Psychologic distress (anxiety and/or depression) was seen in 227 patients (56\%), hypersensitivity (allodynia and/ or hyperalgesia) in 190 patients (47\%), and abnormal transit (accelerated or delayed) in 102 patients (25\%); all 3 of these abnormalities were present in 19 patients (4.7\%), 2 in 150 patients (36.9\%), 1 in 162 patients (39.8\%), and 76

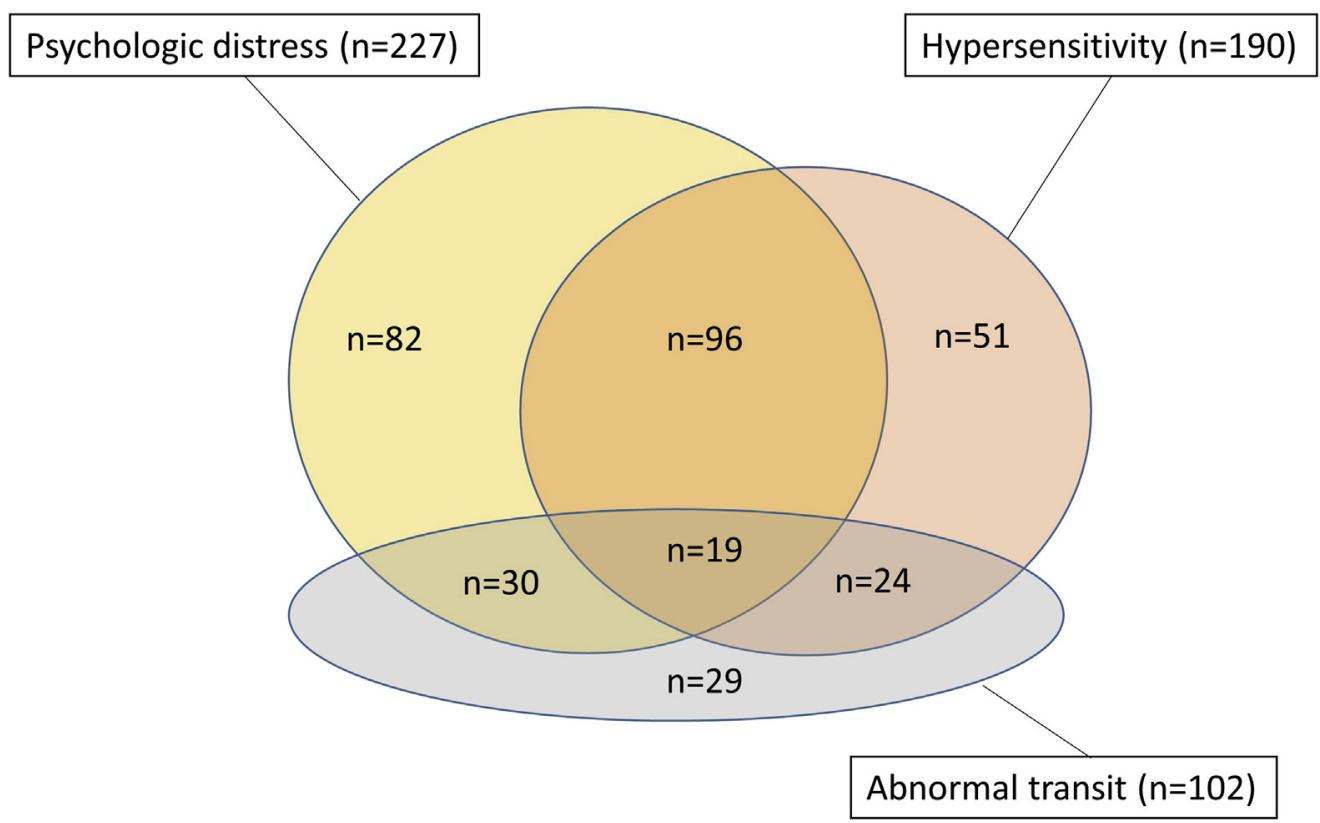

Figure 2. Venn diagram demonstrating the overlap between pathophysiologic factors in our IBS cohort $(n=407)$, using the model with 3 pathophysiologic abnormalities: psychologic distress (anxiety and/or depression), hypersensitivity (allodynia and/or hyperalgesia), and abnormal transit (delayed or accelerated). Seventysix of the patients $(18.7 \%)$ had none of the abnormalities; these patients are not included in the figure. The size of the different areas are not entirely proportional to the number of subjects in each area. 
Table 2. IBS Symptom Severity in Patients With Vs Without Pathophysiological Factors

\begin{tabular}{|c|c|c|c|c|c|c|c|c|c|c|}
\hline & \multicolumn{4}{|c|}{ IBS-SSS $(n=270)$} & \multicolumn{4}{|c|}{ GSRS-IBS $(n=137)$} & \multicolumn{2}{|c|}{ BSF $(n=407)$} \\
\hline & $\begin{array}{c}\text { Pain } \\
\text { intensity }\end{array}$ & $\begin{array}{c}\text { Pain } \\
\text { Frequency }\end{array}$ & Bloating & $\begin{array}{l}\text { Bowel Habit } \\
\text { Dissatis- } \\
\text { faction }\end{array}$ & $\begin{array}{l}\text { Abdominal } \\
\text { pain }\end{array}$ & Bloating & Constipation & Diarrhea & $\begin{array}{l}\text { Stool } \\
\text { Frequency } \\
\text { (stools/day) }\end{array}$ & $\begin{array}{c}\text { Stool } \\
\text { Consistency } \\
\text { (mean } \\
\text { score) }\end{array}$ \\
\hline Allodynia $(n=145)$ & $\begin{array}{c}52 \pm 28 \text { vs. } \\
52 \pm 26 \\
P=.98\end{array}$ & $\begin{array}{c}65 \pm 33 \text { vs. } \\
56 \pm 35 \\
P=.047\end{array}$ & $\begin{array}{c}63 \pm 28 \text { vs. } \\
52 \pm 26 \\
P=.30\end{array}$ & $\begin{array}{l}70 \pm 26 \text { vs. } 71 \pm 24 \\
P=.61\end{array}$ & $\begin{array}{r}4.4 \pm 1.3 \text { vs. } \\
4.1 \pm 1.4 \\
P=.18\end{array}$ & $\begin{array}{c}4.9 \pm 1.2 \text { vs. } \\
4.6 \pm 1.4 \\
P=.15\end{array}$ & $\begin{aligned} 3.0 \pm 1.7 \text { vs. } \\
3.0 \pm 1.7 \\
P=.81\end{aligned}$ & $\begin{aligned} 3.8 \pm 1.3 \text { vs. } \\
3.6 \pm 1.4 \\
P=.42\end{aligned}$ & $\begin{array}{c}2.2 \pm 1.4 \text { vs. } \\
1.7 \pm 1.1 \\
P<.0001 \#\end{array}$ & $\begin{array}{r}4.2 \pm 1.1 \text { vs. } \\
4.1 \pm 1.1 \\
P=0.23\end{array}$ \\
\hline $\begin{array}{l}\text { Hyperalgesia } \\
\quad(n=86)\end{array}$ & $\begin{array}{c}59 \pm 26 \text { vs. } \\
50 \pm 27 \\
P=.012 \#\end{array}$ & $\begin{array}{r}73 \pm 32 \text { vs. } \\
55 \pm 35 \\
P<.0001 \#\end{array}$ & $\begin{array}{c}66 \pm 25 \text { vs. } \\
59 \pm 27 \\
P=.084\end{array}$ & $\begin{array}{l}73 \pm 26 \text { vs. } 70 \pm 25 \\
P=.39\end{array}$ & $\begin{array}{c}4.6 \pm 1.3 \text { vs. } \\
4.0 \pm 1.4 \\
P=.0030 \#\end{array}$ & $\begin{array}{c}4.8 \pm 1.3 \text { vs. } \\
4.6 \pm 1.3 \\
P=.25\end{array}$ & $\begin{aligned} 3.0 \pm 1.7 \text { vs. } \\
2.9 \pm 1.7 \\
P=.75\end{aligned}$ & $\begin{aligned} 3.6 \pm 1.2 \text { vs. } \\
3.7 \pm 1.2 \\
P=.70\end{aligned}$ & $\begin{array}{l}1.8 \pm 1.2 \text { vs. } \\
1.9 \pm 1.2 \\
P=.75\end{array}$ & $\begin{array}{c}4.4 \pm 1.2 \text { vs. } \\
4.1 \pm 1.1 \\
P=.020 \#\end{array}$ \\
\hline $\begin{array}{l}\text { Accelerated } \\
\text { transit }(n=73)\end{array}$ & $\begin{array}{c}52 \pm 30 \text { vs. } \\
52 \pm 26 \\
P=.98\end{array}$ & $\begin{array}{c}63 \pm 37 \text { vs. } \\
58 \pm 34 \\
P=.36\end{array}$ & $\begin{array}{c}55 \pm 30 \text { vs. } \\
62 \pm 26 \\
P=.092\end{array}$ & $\begin{array}{l}77 \pm 26 \text { vs. } 69 \pm 25 \\
P=.063\end{array}$ & $\begin{array}{r}4.3 \pm 1.5 \text { vs. } \\
4.1 \pm 1.4 \\
P=.61\end{array}$ & $\begin{array}{c}\text { (4.3 } \pm 1.4 \text { vs. } \\
4.7 \pm 1.3) \\
P=.032 \#\end{array}$ & $\begin{array}{c}(2.2 \pm 1.5 \text { vs. } \\
3.1 \pm 1.7) \\
P<.0001 \#\end{array}$ & $\begin{array}{c}4.2 \pm 1.4 \text { vs. } \\
3.5 \pm 1.3 \\
P<.0001 \#\end{array}$ & $\begin{array}{c}2.4 \pm 1.7 \text { vs. } \\
1.7 \pm 1.1 \\
P=.0020 \#\end{array}$ & $\begin{array}{c}4.9 \pm 1.0 \text { vs. } \\
4.0 \pm 1.1 \\
P<.0001 \#\end{array}$ \\
\hline $\begin{array}{l}\text { Delayed transit } \\
\qquad(n=29)\end{array}$ & $\begin{array}{c}45 \pm 29 \text { vs. } \\
52 \pm 27 \\
P=.25\end{array}$ & $\begin{array}{c}43 \pm 32 \text { vs. } \\
\quad 60 \pm 35 \\
P=.06\end{array}$ & $\begin{array}{c}55 \pm 31 \text { vs. } \\
\quad 61 \pm 27 \\
P=.42\end{array}$ & $\begin{array}{l}68 \pm 29 \text { vs. } 71 \pm 25 \\
P=.67\end{array}$ & $\begin{array}{c}4.0 \pm 1.6 \text { vs. } \\
4.2 \pm 1.4 \\
P=.51\end{array}$ & $\begin{array}{c}4.2 \pm 1.3 \text { vs. } \\
4.7 \pm 1.3 \\
P=.087\end{array}$ & $\begin{array}{c}3.6 \pm 1.4 \text { vs. } \\
2.9 \pm 1.7 \\
P=.041\end{array}$ & $\begin{array}{c}(2.3 \pm 0.6 \text { vs. } \\
3.8 \pm 1.3) \\
P<.0001 \#\end{array}$ & $\begin{array}{c}1.2 \pm 0.9 \text { vs. } \\
1.9 \pm 1.2 \\
P=.0090 \#\end{array}$ & $\begin{array}{c}3.1 \pm 31.1 \text { vs. } \\
4.2 \pm 1.1 \\
P<.0001 \#\end{array}$ \\
\hline $\begin{array}{l}\text { Increased } \\
\quad \text { compliance } \\
\quad(n=58)\end{array}$ & $\begin{array}{c}58 \pm 26 \text { vs. } \\
51 \pm 27 \\
P=.083\end{array}$ & $\begin{array}{c}65 \pm 32 \text { vs. } \\
57 \pm 35 \\
P=.13\end{array}$ & $\begin{array}{c}66 \pm 25 \text { vs. } \\
59 \pm 27 \\
P=.11\end{array}$ & $\begin{array}{l}65 \pm 29 \text { vs. } 72 \pm 24 \\
P=.15\end{array}$ & $\begin{array}{r}4.3 \pm 1.3 \text { vs. } \\
4.2 \pm 1.4 \\
P=.57\end{array}$ & $\begin{aligned} 4.8 \pm 1.2 \text { vs. } \\
4.6 \pm 1.4 \\
P=.37\end{aligned}$ & $\begin{aligned} 2.7 \pm 1.7 \text { vs. } & 3.0 \pm 1.7 \\
P= & .39\end{aligned}$ & $\begin{aligned} 4.0 \pm 1.3 \text { vs. } & \\
& 3.6 \pm 1.4 \\
P= & .090\end{aligned}$ & $\begin{array}{l}1.8 \pm 1.5 \text { vs. } \\
1.9 \pm 1.2 \\
P=.93\end{array}$ & $\begin{aligned} 4.3 \pm 1.1 \text { vs. } & 4.1 \pm 1.2 \\
P= & .21\end{aligned}$ \\
\hline $\begin{array}{l}\text { Reduced } \\
\quad \text { compliance } \\
(\mathrm{n}=38)\end{array}$ & $\begin{array}{c}47 \pm 29 \text { vs. } \\
52 \pm 27 \\
P=.33\end{array}$ & $\begin{array}{c}48 \pm 38 \text { vs. } \\
60 \pm 34 \\
P=.13\end{array}$ & $\begin{array}{c}51 \pm 26 \text { vs. } \\
61 \pm 27 \\
P=.072\end{array}$ & $\begin{array}{l}69 \pm 21 \text { vs. } 71 \pm 25 \\
P=.71\end{array}$ & $\begin{aligned} 3.7 \pm 1.4 \text { vs. } \\
4.2 \pm 1.4 \\
P=.12\end{aligned}$ & $\begin{array}{c}4.1 \pm 1.4 \text { vs. } \\
4.7 \pm 1.3 \\
P=.052\end{array}$ & $\begin{aligned} 2.5 \pm 1.8 \text { vs. } \\
3.0 \pm 1.7 \\
P=.18\end{aligned}$ & $\begin{aligned} 3.6 \pm 1.0 \text { vs. } \\
3.7 \pm 1.4 \\
P=.82\end{aligned}$ & $\begin{aligned} & 2.0 \pm 1.0 \text { vs. } \\
& 1.8 \pm 1.3 \\
& P=.44\end{aligned}$ & $\begin{array}{l}4.2 \pm 1.2 \text { vs. } \\
4.1 \pm 1.1 \\
P=.90\end{array}$ \\
\hline Anxiety $(n=211)$ & $\begin{array}{c}55 \pm 27 \text { vs. } \\
49 \pm 27 \\
P=.046\end{array}$ & $\begin{array}{c}62 \pm 33 \text { vs. } \\
54 \pm 36 \\
P=.047\end{array}$ & $\begin{array}{c}64 \pm 27 \text { vs. } \\
57 \pm 28 \\
P=.025 \#\end{array}$ & $\begin{array}{l}73 \pm 24 \text { vs. } 68 \pm 26 \\
P=.08\end{array}$ & $\begin{array}{l}4.6 \pm 1.1 \text { vs. } \\
3.8 \pm 1.5 \\
P<.0001 \#\end{array}$ & $\begin{array}{c}4.9 \pm 1.3 \text { vs. } \\
4.4 \pm 1.3 \\
P=.0010 \#\end{array}$ & $\begin{array}{c}3.3 \pm 1.7 \text { vs. } \\
2.6 \pm 1.7 \\
P=.0020 \#\end{array}$ & $\begin{array}{c}3.8 \pm 1.3 \text { vs. } \\
3.5 \pm 1.4 \\
P=.038\end{array}$ & $\begin{aligned} & 1.8 \pm 1.1 \text { vs. } 1.9 \pm 1.4 \\
& P=.43\end{aligned}$ & $\begin{aligned} 4.1 \pm 1.2 \text { vs. } \\
\quad 4.2 \pm 1.1 \\
P=.62\end{aligned}$ \\
\hline Depression $(n=97)$ & $\begin{array}{r}63 \pm 24 \text { vs. } \\
48 \pm 27 \\
P<.0001 \#\end{array}$ & $\begin{array}{r}68 \pm 33 \text { vs. } \\
55 \pm 35 \\
P=.0080 \#\end{array}$ & $\begin{array}{c}64 \pm 24 \text { vs. } \\
59 \pm 28 \\
P=.17\end{array}$ & $\begin{array}{l}74 \pm 26 \text { vs. } 69 \pm 25 \\
P=.16\end{array}$ & $\begin{array}{c}4.7 \pm 1.2 \text { vs. } \\
4.0 \pm 1.4 \\
P=.0010 \#\end{array}$ & $\begin{aligned} 4.9 \pm 1.2 \text { vs. } \\
4.6 \pm 1.4 \\
P=.089\end{aligned}$ & $\begin{array}{r}3.2 \pm 1.7 \text { vs. } \\
2.9 \pm 1.7 \\
P=.15\end{array}$ & $\begin{array}{r}4.0 \pm 1.4 \text { vs. } \\
3.5 \pm 1.3 \\
P=.014 \#\end{array}$ & $\begin{array}{l}2.0 \pm 1.3 \text { vs. } \\
1.8 \pm 1.2 \\
P=.17\end{array}$ & $\begin{aligned} 4.2 \pm 1.2 \text { vs. } & 4.1 \pm 1.1 \\
P= & .75\end{aligned}$ \\
\hline
\end{tabular}

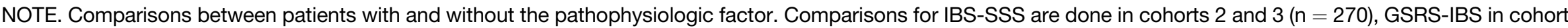

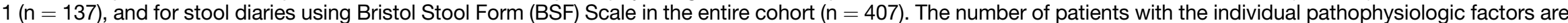

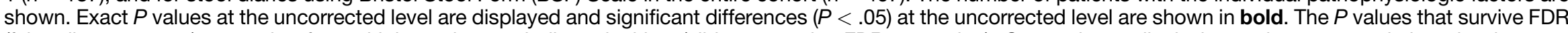

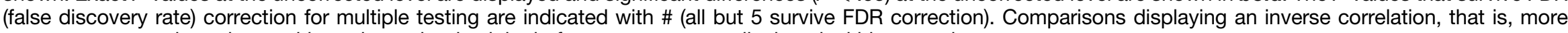
severe symptoms in patients without the pathophysiologic factor present, are displayed within parentheses. 
patients $(18.7 \%)$ had none of the abnormalities. The overlap between these variables can be seen in Figure 2 .

\section{Association Between Number of Pathophysiologic Factors and PROs}

For analysis purposes, the patients were placed in 4 groups based on the number of pathophysiologic abnormalities relevant for IBS symptom severity; none, 1, 2, or 3 or more abnormalities. Symptom severity showed a gradual increase with increasing number of pathophysiologic factors, with medium effect size for IBS symptom severity (Partial $\left.\eta^{2}=0.093 ; P<.0001\right)$ and large effect size for overall somatic symptom severity (Partial $\eta^{2}=0.159 ; P<$ .0001) (Figure 3A; Table 3). There was a gradual decrease in quality of life with increasing number of pathophysiologic factors with medium to large effect sizes for all IBSQOL domains except for the food domain (small effect size) (Figure 3B, Table 3). Separate analyses in the 3 IBS cohorts yielded similar results with medium to large effect sizes for IBS and overall somatic symptom severity, as well as for most of the IBSQOL domains; significant linear trends were seen for all the analyses except for 2 IBSQOL domains in IBS cohort 2 and 1 in IBS cohort 3 (Supplementary Figure 1, Supplementary Table 2).

In a subsequent step we performed the same analyses but removed anxiety and depression, that is, used only 3 pathophysiologic factors (allodynia, hyperalgesia, and abnormal oroanal transit), and placed individuals into 3 groups (none, 1 , or 2 or more abnormalities). For all the PROs except for the IBSQOL emotional subscale, significant linear trends with gradually increasing symptom severity and decreasing IBS-specific quality of life with increasing number of pathophysiologic abnormalities were seen, but the effect sizes were reduced to small (Table 3). When only anxiety and depression were used in the analyses (no, 1, or 2 abnormalities), significant linear trends with gradually increasing symptom severity and decreasing IBS-specific quality of life with increasing number of pathophysiologic abnormalities with medium to large effect sizes were seen (Table 3).

Using the alternative grouping of pathophysiologic factors (psychologic distress, hypersensitivity, and abnormal transit) yielded similar results with significant linear trends, but a tendency toward smaller effect sizes compared with the model with a larger number of pathophysiologic factors
A

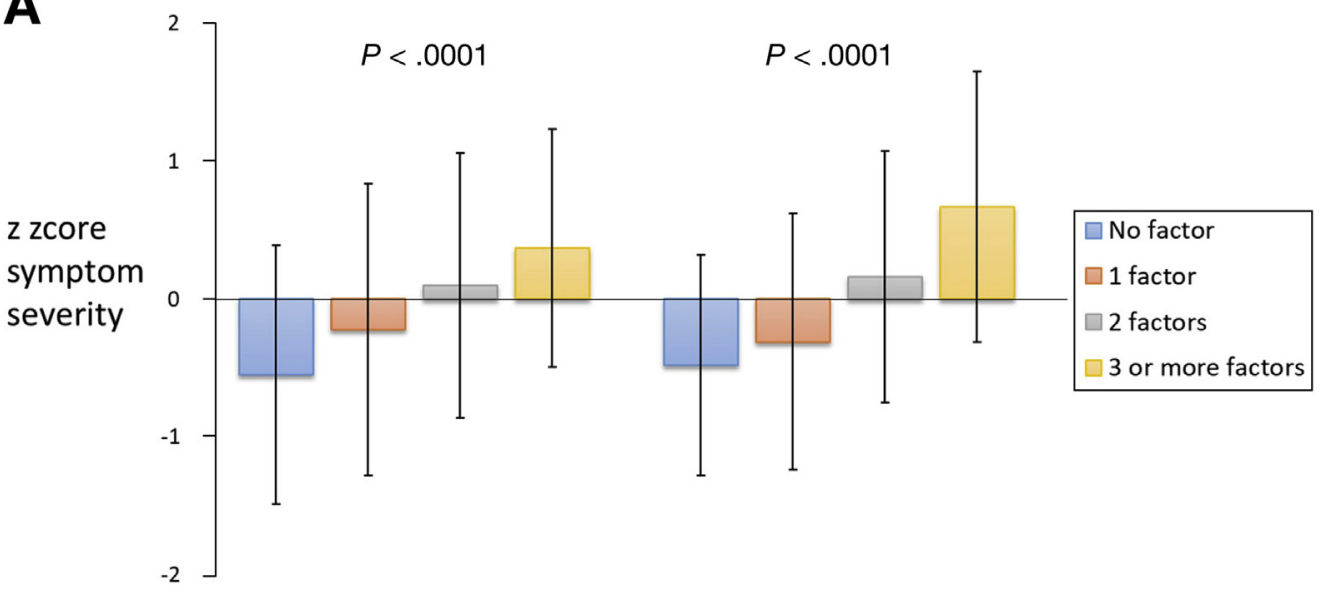

IBS symptom severity Overall somatic symptom severity

B

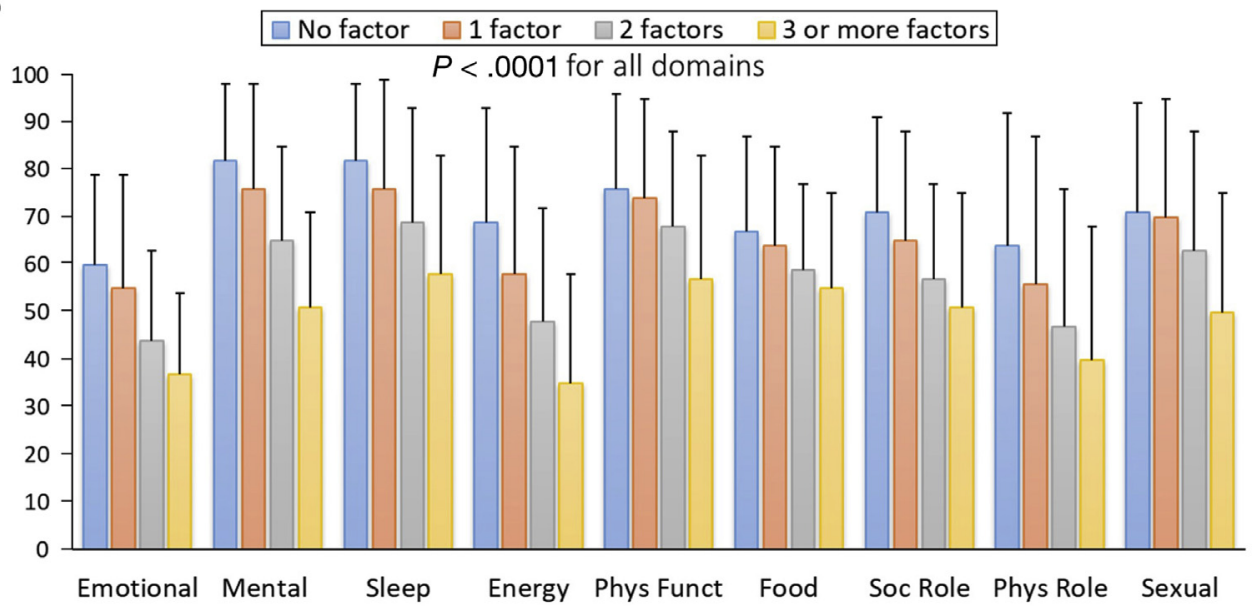

Figure 3. Gradual increase in $(A)$ IBS symptom severity ( $z$ score of IBSSSS and GSRS-IBS total scores) and overall somatic symptom severity $(z$ score of PHQ-15 and Symptom Checklist-90 somatization subscale), and $(B)$ gradual decrease in IBSQOL for all domains with increasing number of pathophysiologic abnormalities. $P$ values from ANOVA with linear trend analysis. 
Table 3.ANOVA With Linear Trend Analyses: Number of Pathophysiologic Factors and Patient-Reported Outcomes

\begin{tabular}{|c|c|c|c|c|c|c|}
\hline & \multicolumn{2}{|c|}{ All factors } & \multicolumn{2}{|c|}{$\begin{array}{c}\text { Allodynia+hyperalgesia } \\
\text { +transit }\end{array}$} & \multicolumn{2}{|c|}{ Anxiety \& depression } \\
\hline & Partial $\eta^{2}$ & $P$ & Partial $\eta^{2}$ & $P$ & Partial $\eta^{2}$ & $P$ \\
\hline IBS sx & $.093^{b}$ & .000 & $.021^{a}$ & .004 & $.107^{b}$ & .000 \\
\hline Somatic sx & $.159^{c}$ & .000 & $.022^{a}$ & .003 & $.197^{c}$ & .000 \\
\hline IBSQOL_Emotional & $.138^{b}$ & .000 & .004 & .223 & $.231^{c}$ & .000 \\
\hline IBSQOL_Mental & $.219^{\circ}$ & .000 & $.012^{a}$ & .041 & $.356^{c}$ & .000 \\
\hline IBSQOL_Sleep & $.108^{b}$ & .000 & $.051^{a}$ & .000 & $.100^{b}$ & .000 \\
\hline IBSQOL_Energy & $.167^{\circ}$ & .000 & $.042^{a}$ & .000 & $.172^{\circ}$ & .000 \\
\hline IBSQOL_PhysFunct & $.075^{b}$ & .000 & $.030^{a}$ & .001 & $.092^{b}$ & .000 \\
\hline IBSQOL_Food & $.045^{a}$ & .000 & $.015^{a}$ & .018 & $.045^{a}$ & .000 \\
\hline IBSQOL_SocialRole & $.093^{b}$ & .000 & $.014^{a}$ & .021 & $.126^{b}$ & .000 \\
\hline IBSQOL_PhysicalRole & $.074^{b}$ & .000 & $.012^{a}$ & .032 & $.098^{b}$ & .000 \\
\hline IBSQOL_Sexual & $.075^{b}$ & .000 & $.034^{a}$ & .002 & $.077^{b}$ & .000 \\
\hline
\end{tabular}

NOTE. Significance indicated in bold.

The effect size (Partial $\left.\eta^{2}\right)$ is indicated as follows:

${ }^{a}$ Small $(0.01-0.05)$.

${ }^{b}$ Medium (0.06-0.13).

${ }^{c}$ Large $(\geq 0.14)$.

(Table 4). Furthermore, multivariate linear regression models with IBS symptom severity and overall somatic symptom severity as dependent variables, and psychologic distress, hypersensitivity, and abnormal transit as dichotomous independent variables, demonstrated that hypersensitivity and psychologic distress were both independently associated with the outcome variables (Supplementary Tables 3 and 4).

\section{Discussion}

In this study, we have demonstrated in a large group of patients with IBS that well-defined pathophysiologic factors that are putatively involved in symptom generation in patients with IBS seem to influence relevant patient-reported outcome variables in a cumulative fashion. Specifically, visceral hypersensitivity, that is, allodynia and hyperalgesia, abnormal oroanal or colonic transit, and psychologic factors were all associated with GI symptom severity in IBS, and demonstrated a cumulative effect on the severity of GI and non-GI symptoms, as well as on quality of life impairment in IBS. Our findings fit well with the current view on the important role of gut-brain interactions (ie, reciprocal communication between the gut and the brain) in symptoms in patients with functional GI disorders such as IBS. ${ }^{1,30}$

All of the pathophysiologic factors we included in this study have been shown in previous studies to be associated with, and hence of potential relevance for, severity of symptoms in patients with IBS. ${ }^{5-7,9,21,22,25-27,29}$ Regarding the association with IBS symptoms, previous studies have indicated that colonic motility (mostly measured as colonic or oroanal transit time) is more clearly associated with the abnormal bowel habits in IBS, ${ }^{5,9,25-27}$ whereas visceral hypersensitivity seems to be more clearly associated with abdominal pain. ${ }^{6,7}$ Only psychologic distress and visceral hypersensitivity have demonstrated an association with overall IBS symptom severity. ${ }^{7,21,22,29}$ Our analyses confirm these findings with a general pattern of association between motility/transit and bowel habit abnormalities, visceral hypersensitivity and abdominal pain, and psychologic factors being of importance for the overall IBS symptomatology. However, our study extends beyond the previous studies, as it also demonstrates a striking cumulative effect of these factors on the overall IBS symptomatology, and also on overall somatic symptom severity and disease-specific quality of life, highlighting the importance of considering and assessing combinations of pathophysiologic factors in IBS and pointing toward the importance of the gut-brain

Table 4. ANOVA With Linear Trend Analyses: Number of Pathophysiologic Factors Using the Alternative Grouping, Psychologic Distress, Hypersensitivity and Abnormal Transit, and Patient-Reported Outcomes

\begin{tabular}{lcc}
\hline & \multicolumn{2}{c}{$\begin{array}{c}\text { Psychologic distress, } \\
\text { hypersensitivity }+ \text { abnormal transit }\end{array}$} \\
\cline { 2 - 3 } & Partial $\eta^{2}$ & p-value \\
\hline IBS sx & $.066^{b}$ & .000 \\
Somatic sx & $.106^{b}$ & .000 \\
IBSQOL_Emotional & $.111^{b}$ & .000 \\
IBSQOL_Mental & $.148^{c}$ & .000 \\
IBSQOL_Sleep & $.064^{b}$ & .000 \\
IBSQOL_Energy & $.122^{b}$ & .000 \\
IBSQOL_PhysFunct & $.061^{b}$ & .000 \\
IBSQOL_Food &. $\mathbf{0 4 1 ^ { a }}$ & .000 \\
IBSQOL_SocialRole &. $\mathbf{0 7 4}$ & .000 \\
IBSQOL_PhysicalRole &. $\mathbf{0 4 8 ^ { a }}$ & .000 \\
IBSQOL_Sexual &. $\mathbf{0 7 7 ^ { b }}$ & \\
& &
\end{tabular}

The effect size (Partial $\left.\eta^{2}\right)$ is indicated as follows:

${ }^{a}$ Small (0.01-0.05).

${ }^{b}$ Medium (0.06-0.13).

${ }^{c}$ Large $(\geq 0.14)$. 
axis in this process. ${ }^{30}$ Our findings also seem robust, as different approaches to grouping the pathophysiologic factors yielded similar results, and the findings in the overall IBS cohort were congruent with findings in the 3 individual IBS cohorts analyzed separately. Moreover, even though anxiety and depression were the strongest factors when it comes to the overall symptomatology in our IBS sample, removing these factors from the analyses did not eliminate the significant linear trend between the number of pathophysiologic factors and the severity of PROs, although the effect sizes were reduced. However, even though our study indicates that abnormal transit seems to contribute the least of the pathophysiologic factors to the PROs, it is of importance because it contributed significantly to several of the models tested. This indicates that the cumulative effect seen is not solely explained by the psycholologic factors included in our study.

Current therapeutic options in IBS have limited efficacy. Positive effects on symptoms are achieved in only subsets of patients, and in most patients the available therapies only produce partial improvement in symptoms and rarely lead to complete symptom resolution., ${ }^{2,3}$ This fits well with the findings in this study, in which many patients demonstrated the presence of several pathophysiologic abnormalities, with a complex overlap pattern, and greater number of these abnormalities associated stepwise with greater symptom severity. Hence, targeting only one of these key pathophysiologic factors is unlikely to lead to complete symptom resolution, even if that abnormality is present in the individual patient. Based on our findings, it is tempting to speculate that combining therapies that target different levels of the gut-brain axis and different pathophysiologic factors simultaneously might lead to improved treatment outcomes. However, that hypothesis of course needs to be tested in prospective treatment trials. Furthermore, in today's clinical setting, the symptom profile is used to guide therapy, ${ }^{2}$ and knowledge about the pathophysiology in the individual patients is rarely available, which probably further reduces the likelihood of treatment success. In the future, more personalized treatment approaches based on the underlying pathophysiology, including combinations of abnormalities, may optimize the treatment approach to these subtypes of patients with IBS defined on more complex merits than bowel habits and dominant symptoms. ${ }^{2}$

Our study had several strengths. We studied a wellcharacterized and large group of patients with IBS evaluated at an expert center for functional GI disorders and used the current state of the art methodology for assessment of pathophysiology in IBS. Moreover all patients were studied in a single center using uniform study methodology and design, except for use of different questionnaires and rectal barostat protocols during different time periods. However, the slight differences in the methodology across the cohorts were of minor importance because the main findings in the overall cohort were also seen in the 3 individual IBS cohorts. Moreover, being evaluated at an expert center can be considered as a weakness as well, because the findings might not be generalizable to patients with IBS in the community. We believe that this a minor issue in this cohort because almost all patients were referred from primary care or through self-referral specifically for general advice regarding treatment and investigations, but were otherwise managed in primary care, just as community patients with IBS. The update of diagnostic criteria for IBS over the course of this study (ie, Rome II to Rome III) ${ }^{36,37}$ also could be viewed as a weakness, but because the 3 cohorts were similar regarding demographic factors as well as GI, overall somatic, and psychologic symptom severity, we consider it unlikely that this had any relevant impact on our findings. Moreover, we focused on a limited number of pathophysiology factors, and there are of course other factors of potential relevance in IBS that were not included in this study, such as variations in the gut microenvironment. ${ }^{13-17} \mathrm{We}$ also used psychologic symptoms as a proxy measure for abnormal brain function, which may not be entirely accurate, but for the purpose of the analyses in this study we consider this approach to be acceptable.

In conclusion, we have demonstrated in this study that visceral hypersensitivity, that is, allodynia and hyperalgesia, abnormal colonic or oroanal transit, and psychologic factors, are all associated with GI symptom severity in IBS. These factors seem to have a cumulative effect on GI and overall somatic symptoms, as well as on quality of life in IBS, and therefore constitute relevant treatment targets. Future studies should assess whether other pathophysiologic factors, such as abnormal gut microenvironment, can add to this model and explain even more of the variance in the symptomatology in patients with IBS and interact with the pathophysiologic factors investigated in this study. Moreover, treatment studies targeting multiple pathophysiologic factors simultaneously in patients with IBS with combinations of these abnormalities should be performed to assess if this approach leads to greater therapeutic impact on symptoms than current treatment approaches targeting predominant symptoms without specific knowledge about the underlying pathophysiology in the individual patient.

\section{Supplementary Material}

Note: To access the supplementary material accompanying this article, visit the online version of Gastroenterology at www.gastrojournal.org, and at https://doi.org/10.1053/ j.gastro.2019.04.019.

\section{References}

1. Lacy BE, Mearin F, Chang L, et al. Bowel disorders. Gastroenterology 2016;150:1393-1407.

2. Simren M, Tack J. New treatments and therapeutic targets for IBS and other functional bowel disorders. Nat Rev Gastroenterol Hepatol 2018;15:589-605.

3. Simren M, Tornblom H, Palsson OS, et al. Management of the multiple symptoms of irritable bowel syndrome. Lancet Gastroenterol Hepatol 2017;2:112-122.

4. Enck P, Aziz Q, Barbara G, et al. Irritable bowel syndrome. Nat Rev Dis Primers 2016;2:16014.

5. Camilleri M, McKinzie S, Busciglio I, et al. Prospective study of motor, sensory, psychologic, and autonomic 
functions in patients with irritable bowel syndrome. Clin Gastroenterol Hepatol 2008;6:772-781.

6. Kanazawa M, Palsson OS, Thiwan SI, et al. Contributions of pain sensitivity and colonic motility to IBS symptom severity and predominant bowel habits. Am J Gastroenterol 2008;103:2550-2561.

7. Posserud I, Syrous A, Lindstrom L, et al. Altered rectal perception in irritable bowel syndrome is associated with symptom severity. Gastroenterology 2007;133:11131123.

8. Whitehead WE, Engel BT, Schuster MM. Irritable bowel syndrome: physiological and psychological differences between diarrhea-predominant and constipationpredominant patients. Dig Dis Sci 1980;25:404-413.

9. Tornblom H, Van Oudenhove L, Sadik R, et al. Colonic transit time and IBS symptoms: what's the link? Am J Gastroenterol 2012;107:754-760.

10. Elsenbruch S, Rosenberger C, Bingel U, et al. Patients with irritable bowel syndrome have altered emotional modulation of neural responses to visceral stimuli. Gastroenterology 2010;139:1310-1319.

11. Tillisch K, Mayer EA, Labus JS. Quantitative metaanalysis identifies brain regions activated during rectal distension in irritable bowel syndrome. Gastroenterology 2011;140:91-100.

12. Van Oudenhove L, Crowell MD, Drossman DA, et al. Biopsychosocial aspects of functional gastrointestinal disorders. Gastroenterology 2016;150:1355-1367.

13. Barbara G, Feinle-Bisset C, Ghoshal UC, et al. The intestinal microenvironment and functional gastrointestinal disorders. Gastroenterology 2016;150:1305-1318.

14. Bashashati M, Moossavi S, Cremon C, et al. Colonic immune cells in irritable bowel syndrome: a systematic review and meta-analysis. Neurogastroenterol Motil 2018;30:e13192.

15. Bednarska O, Walter SA, Casado-Bedmar M, et al. Vasoactive intestinal polypeptide and mast cells regulate increased passage of colonic bacteria in patients with irritable bowel syndrome. Gastroenterology 2017; 153:948-960.e3.

16. Bennet SM, Polster A, Tornblom H, et al. Global cytokine profiles and association with clinical characteristics in patients with irritable bowel syndrome. Am J Gastroenterol 2016;111:1165-1176.

17. Tap J, Derrien M, Tornblom H, et al. Identification of an intestinal microbiota signature associated with severity of irritable bowel syndrome. Gastroenterology 2017; 152:111-123.e8.

18. Keszthelyi D, Troost FJ, Masclee AA. Irritable bowel syndrome: methods, mechanisms, and pathophysiology. Methods to assess visceral hypersensitivity in irritable bowel syndrome. Am J Physiol Gastrointest Liver Physiol 2012;303:G141-G154.

19. Bouin M, Plourde V, Boivin M, et al. Rectal distention testing in patients with irritable bowel syndrome: sensitivity, specificity, and predictive values of pain sensory thresholds. Gastroenterology 2002;122:1771-1777.

20. Tornblom H, Van Oudenhove L, Tack J, et al. Interaction between preprandial and postprandial rectal sensory and motor abnormalities in IBS. Gut 2014;63:1441-1449.
21. van der Veek PP, Van Rood YR, Masclee AA. Symptom severity but not psychopathology predicts visceral hypersensitivity in irritable bowel syndrome. Clin Gastroenterol Hepatol 2008;6:321-328.

22. Simren M, Tornblom H, Palsson OS, et al. Visceral hypersensitivity is associated with Gl symptom severity in functional Gl disorders: consistent findings from five different patient cohorts. Gut 2018;67:255-262.

23. Chey WY, Jin $\mathrm{HO}$, Lee $\mathrm{MH}$, et al. Colonic motility abnormality in patients with irritable bowel syndrome exhibiting abdominal pain and diarrhea. Am J Gastroenterol 2001;96:1499-1506.

24. Clemens CH, Samsom M, Van Berge Henegouwen GP, et al. Abnormalities of left colonic motility in ambulant nonconstipated patients with irritable bowel syndrome. Dig Dis Sci 2003;48:74-82.

25. Manabe N, Wong BS, Camilleri M, et al. Lower functional gastrointestinal disorders: evidence of abnormal colonic transit in a 287 patient cohort. Neurogastroenterol Motil 2010;22:293-e82.

26. Saad RJ, Rao SS, Koch KL, et al. Do stool form and frequency correlate with whole-gut and colonic transit? Results from a multicenter study in constipated individuals and healthy controls. Am J Gastroenterol 2010; 105:403-411.

27. Sadik R, Bjornsson E, Simren M. The relationship between symptoms, body mass index, gastrointestinal transit and stool frequency in patients with irritable bowel syndrome. Eur J Gastroenterol Hepatol 2010;22:102108.

28. Lee C, Doo E, Choi JM, et al. The increased level of depression and anxiety in irritable bowel syndrome patients compared with healthy controls: systematic review and meta-analysis. J Neurogastroenterol Motil 2017; 23:349-362.

29. Pinto-Sanchez MI, Ford AC, Avila CA, et al. Anxiety and depression increase in a stepwise manner in parallel with multiple FGIDs and symptom severity and frequency. Am J Gastroenterol 2015;110:1038-1048.

30. Drossman DA, Hasler WL. Rome IV-functional GI disorders: disorders of gut-brain interaction. Gastroenterology 2016;150:1257-1261.

31. Elsenbruch S, Rosenberger C, Enck P, et al. Affective disturbances modulate the neural processing of visceral pain stimuli in irritable bowel syndrome: an fMRI study. Gut 2010;59:489-495.

32. Larsson MB, Tillisch $\mathrm{K}$, Craig AD, et al. Brain responses to visceral stimuli reflect visceral sensitivity thresholds in patients with irritable bowel syndrome. Gastroenterology 2012;142:463-472.e3.

33. Dorn SD, Palsson OS, Thiwan SI, et al. Increased colonic pain sensitivity in irritable bowel syndrome is the result of an increased tendency to report pain rather than increased neurosensory sensitivity. Gut 2007;56:12021209.

34. Gorard DA, Gomborone JE, Libby GW, et al. Intestinal transit in anxiety and depression. Gut 1996;39:551-555.

35. Latimer P, Sarna S, Campbell D, et al. Colonic motor and myoelectrical activity: a comparative study of normal subjects, psychoneurotic patients, and patients with 
irritable bowel syndrome. Gastroenterology 1981; 80:893-901.

36. Thompson WG, Longstreth GF, Drossman DA, et al. Functional bowel disorders and functional abdominal pain. Gut 1999;45(Suppl 2):||43-I|47.

37. Longstreth GF, Thompson WG, Chey WD, et al. Functional bowel disorders. Gastroenterology 2006; 130:1480-1491.

38. Bohn L, Storsrud S, Tornblom H, et al. Self-reported food-related gastrointestinal symptoms in IBS are common and associated with more severe symptoms and reduced quality of life. Am J Gastroenterol 2013; 108:634-641.

39. Jeffery IB, O'Toole PW, Ohman L, et al. An irritable bowel syndrome subtype defined by species-specific alterations in faecal microbiota. Gut 2012;61:997-1006.

40. Ohman L, Isaksson S, Lindmark AC, et al. T-cell activation in patients with irritable bowel syndrome. Am J Gastroenterol 2009;104:1205-1212.

41. Ohman L, Isaksson S, Lundgren A, et al. A controlled study of colonic immune activity and beta7 + blood $T$ lymphocytes in patients with irritable bowel syndrome. Clin Gastroenterol Hepatol 2005;3:980-986.

42. Ohman L, Lindmark AC, Isaksson S, et al. B-cell activation in patients with irritable bowel syndrome (IBS). Neurogastroenterol Motil 2009;21:644-650.e27.

43. Ohman L, Stridsberg M, Isaksson S, et al. Altered levels of fecal chromogranins and secretogranins in IBS: relevance for pathophysiology and symptoms? Am J Gastroenterol 2012;107:440-447.

44. Polster A, Friberg P, Gunterberg V, et al. Heart rate variability characteristics of patients with irritable bowel syndrome and associations with symptoms. Neurogastroenterol Motil 2018;30:e13320.

45. Wilpart K, Tornblom H, Svedlund J, et al. Coping skills are associated with gastrointestinal symptom severity and somatization in patients with irritable bowel syndrome. Clin Gastroenterol Hepatol 2017;15:1565-1571. e3.

46. Abrahamsson H, Antov S. Accuracy in assessment of colonic transit time with particles: how many markers should be used? Neurogastroenterol Motil 2010; 22:1164-1169.

47. Sadik R, Abrahamsson H, Stotzer PO. Gender differences in gut transit shown with a newly developed radiological procedure. Scand J Gastroenterol 2003; 38:36-42.

48. Sadik R, Stotzer PO, Simren M, et al. Gastrointestinal transit abnormalities are frequently detected in patients with unexplained Gl symptoms at a tertiary centre. Neurogastroenterol Motil 2008;20:197-205.

49. Cremonini F, Houghton LA, Camilleri M, et al. Barostat testing of rectal sensation and compliance in humans: comparison of results across two centres and overall reproducibility. Neurogastroenterol Motil 2005;17:810820.

50. Bjelland I, Dahl AA, Haug TT, et al. The validity of the Hospital Anxiety and Depression Scale. An updated literature review. J Psychosom Res 2002;52:69-77.

51. Zigmond AS, Snaith RP. The hospital anxiety and depression scale. Acta Psychiatr Scand 1983;67:361370.

52. Wiklund IK, Fullerton S, Hawkey CJ, et al. An irritable bowel syndrome-specific symptom questionnaire: development and validation. Scand J Gastroenterol 2003;38:947-954.

53. Francis CY, Morris J, Whorwell PJ. The irritable bowel severity scoring system: a simple method of monitoring irritable bowel syndrome and its progress. Aliment Pharmacol Ther 1997;11:395-402.

54. Lewis SJ, Heaton KW. Stool form scale as a useful guide to intestinal transit time. Scand J Gastroenterol 1997; 32:920-924.

55. Derogatis LR, Rickels K, Rock AF. The SCL-90 and the MMPI: a step in the validation of a new self-report scale. Br J Psychiatry 1976;128:280-289.

56. Kroenke K, Spitzer RL, Williams JB. The PHQ-15: validity of a new measure for evaluating the severity of somatic symptoms. Psychosom Med 2002;64:258-266.

57. Hahn BA, Kirchdoerfer LJ, Fullerton S, et al. Evaluation of a new quality of life questionnaire for patients with irritable bowel syndrome. Aliment Pharmacol Ther 1997; 11:547-552.

58. Pinhas M, Tzelgov J, Ganor-Stern D. Estimating linear effects in ANOVA designs: the easy way. Behav Res Methods 2012;44:788-794.

59. Cohen J. Statistical power analysis for the behavioral sciencies. Abingdon, United Kingdom: Routledge, 1988.

\section{Received December 28, 2018. Accepted April 17, 2019.}

\section{Reprint requests}

Address requests for reprints to: Magnus Simrén, MD, PhD, Department of Internal Medicine and Clinical Nutrition, Institute of Medicine, Sahlgrenska Academy, University of Gothenburg, 40530 Gothenburg, Sweden. e-mail: magnus.simren@medicine.gu.se; fax: +46 317412917.

\section{Acknowledgments}

Author contributions: MS: Principal investigator; study concept; patient inclusion and study conduct; first manuscript draft; statistical analyses; study design; interpretation of results; critical revision of manuscript. HT: Patient inclusion and study conduct; study design; interpretation of results; critical revision of manuscript JT: Study concept; study design; interpretation of results; critical revision of manuscript. LVO, OSP, WEW: Study design, interpretation of results and critical revision of manuscript.

\section{Conflicts of interest}

The authors disclose no conflicts.

\section{Funding}

This study was supported by the Swedish Medical Research Council (grants 13409, 21691, and 21692), AFA Insurance, by the Faculty of Medicine, University of Gothenburg, and by unrestricted grants from Ferring Pharmaceuticals and Glycom A/S. 
A

IBS Cohort $1(n=137)$

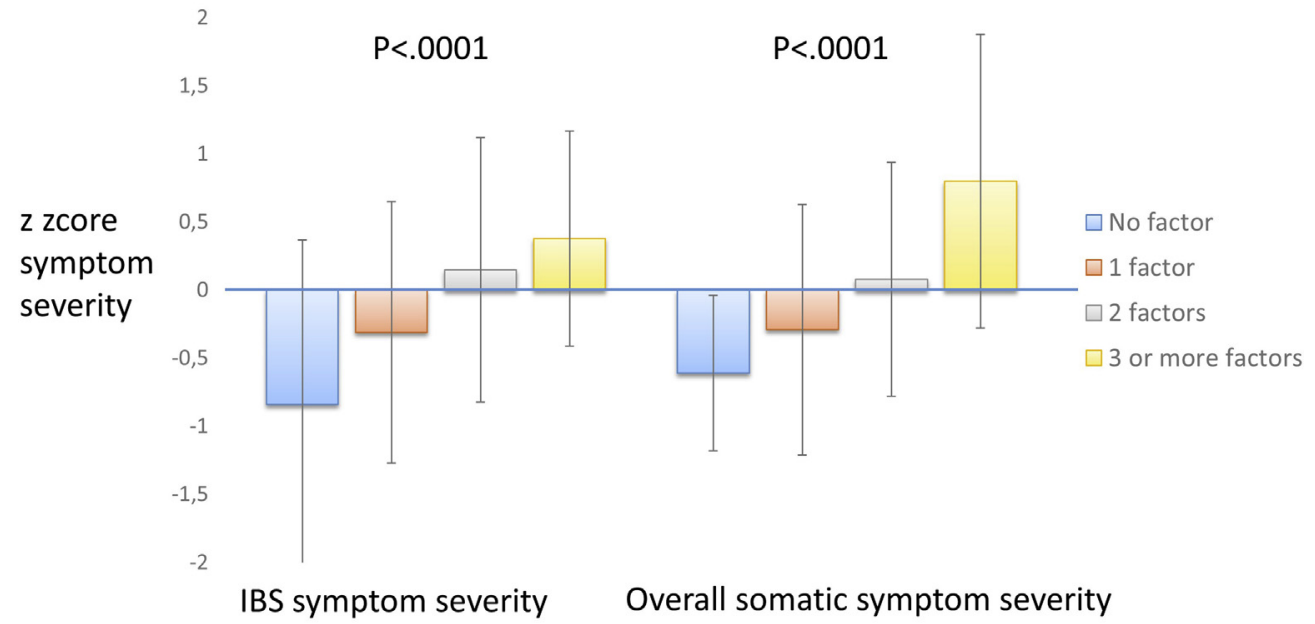

B

IBS Cohort $2(n=128)$

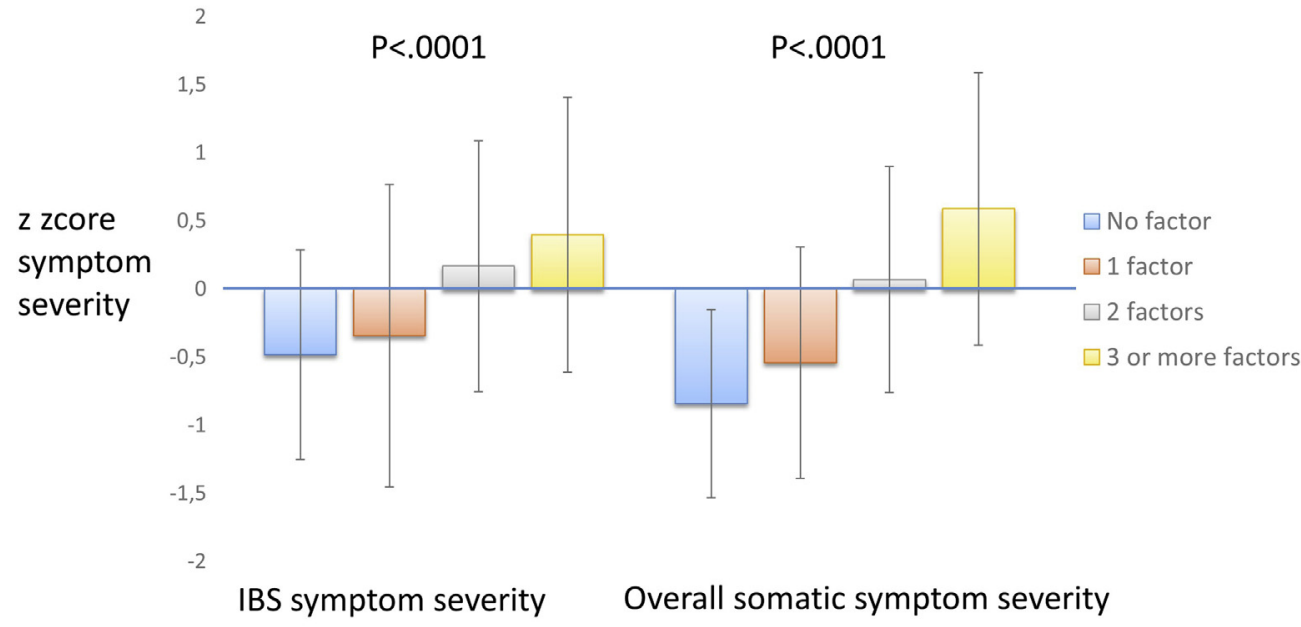

C

IBS Cohort $3(n=142)$

$\mathrm{P}=.003$

$\mathrm{P}<.0001$

Supplementary Figures 1. Gradual increase in IBS symptom severity ( $z$ score of IBS-SSS and GSRS-IBS total scores) and overall somatic symptom severity ( $z$ score of PHQ-15 and Symptom Checklist-90 somatization subscale) with increasing number of pathophysiologic abnormalities in all IBS cohorts $(A-C)$. $P$ values from ANOVA with linear trend analysis.

z zcore

symptom 0,5 severity

1,5

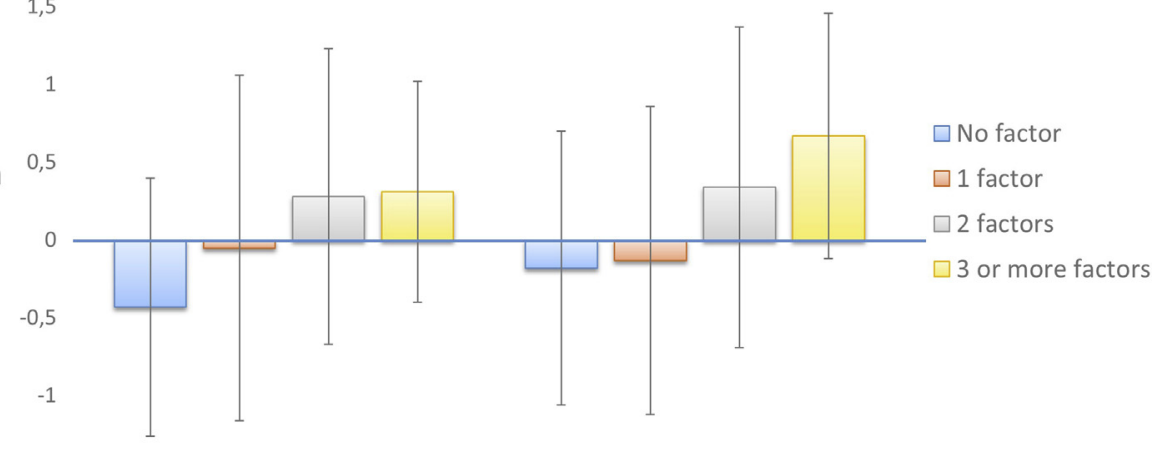

$-1,5$

IBS symptom severity Overall somatic symptom severity 
Supplementary Table 1.Pathophysiologic Measures and Patient-Reported Outcomes Among IBS Subtypes

\begin{tabular}{|c|c|c|c|c|}
\hline & IBS-C (n = 99) & IBS-D (n = 145) & IBSnonCnonD $(n=163)$ & $P^{a}$ \\
\hline Age, $y$, mean $\pm \mathrm{SD}$ & $36.2 \pm 11.3$ & $36.9 \pm 12.1$ & $35.3 \pm 12.4$ & .51 \\
\hline Gender, female/male & $81 / 18$ & $103 / 42$ & $117 / 46$ & .14 \\
\hline HAD anxiety & $8.8 \pm 4.6$ & $8.3 \pm 4.8$ & $7.6 \pm 4.2$ & .08 \\
\hline HAD depression & $5.7 \pm 4.0$ & $5.5 \pm 3.8$ & $4.6 \pm 3.2$ & .02 \\
\hline$Z$ score somatic symptom severity & $0.1 \pm 1.0$ & $0.1 \pm 1.0$ & $-0.1 \pm 1.0$ & .06 \\
\hline$Z$ score IBS symptom severity & $0.0 \pm 0.9$ & $0.0 \pm 1.1$ & $-0.2 \pm 1.0$ & .34 \\
\hline Oroanal transit time, $d$ & $2.2 \pm 1.3$ & $1.2 \pm 0.7$ & $1.5 \pm 0.9$ & .000 \\
\hline Rectal pain threshold, $\mathrm{mm} \mathrm{Hg}$ (protocol 1) & $32 \pm 12$ & $32 \pm 13$ & $33 \pm 13$ & .82 \\
\hline Rectal pain intensity, $\mathrm{mm}$ (at pain threshold; protocol 1) & $35 \pm 22$ & $35 \pm 25$ & $31 \pm 22$ & .48 \\
\hline Rectal pain threshold, $\mathrm{mm} \mathrm{Hg}$ (protocol 2) & $27 \pm 9$ & $28 \pm 9$ & $27 \pm 8$ & .90 \\
\hline Rectal pain intensity, $\mathrm{mm}$ (at $24 \mathrm{~mm} \mathrm{Hg}$; protocol 1) & $55 \pm 32$ & $47 \pm 32$ & $44 \pm 33$ & .29 \\
\hline
\end{tabular}

IBS-C, constipation-predominant IBS; IBS-D, diarrhea-predominant IBS; IBSnonCnonD, nonconstipation or diarrheapredominant IBS, hence patients with IBS mixed/alternating type and unsubtyped IBS; SD, standard deviation.

${ }^{a}$ ANOVA or $\chi^{2}$ test among the 3 different cohorts.

Supplementary Table 2. ANOVA With Linear Trend Analyses: Number of Pathophysiologic Factors and Patient-Reported Outcomes in the 3 IBS Cohorts

\begin{tabular}{|c|c|c|c|c|c|c|}
\hline & \multicolumn{2}{|c|}{ IBS cohort $1(n=137)$} & \multicolumn{2}{|c|}{ IBS cohort $2(n=128)$} & \multicolumn{2}{|c|}{ IBS cohort $3(n=142)$} \\
\hline & Partial $\eta^{2}$ & $P$ & Partial $\eta^{2}$ & $P$ & Partial $\eta^{2}$ & $P$ \\
\hline IBS sx & $.128^{b}$ & .000 & $.100^{b}$ & .000 & $.061^{b}$ & .003 \\
\hline Somatic sx & $.203^{c}$ & .000 & $.256^{c}$ & .000 & $.085^{b}$ & .000 \\
\hline IBSQOL_Emotional & $.174^{C}$ & .000 & $.173^{C}$ & .000 & $.090^{b}$ & .001 \\
\hline IBSQOL_Mental & $.226^{c}$ & .000 & $.319^{c}$ & .000 & $.149^{\circ}$ & .000 \\
\hline IBSQOL_Sleep & $.109^{b}$ & .000 & $.154^{c}$ & .000 & $.059^{a}$ & .000 \\
\hline IBSQOL_Energy & $.198^{C}$ & .000 & $.244^{c}$ & .000 & $.082^{b}$ & .001 \\
\hline IBSQOL_PhysFunct & $.119^{b}$ & .000 & .018 & .14 & $.103^{b}$ & .000 \\
\hline IBSQOL_Food & $.100^{b}$ & .000 & $.076^{b}$ & .002 & .005 & .41 \\
\hline IBSQOL_SocialRole & $.147^{\circ}$ & .000 & $.069^{b}$ & .003 & $.043^{a}$ & .019 \\
\hline IBSQOL_PhysicalRole & $.109^{b}$ & .000 & $.054^{a}$ & .010 & $.047^{a}$ & .014 \\
\hline IBSQOL_Sexual & $.064^{b}$ & .017 & .043 & .058 & $.147^{b}$ & .000 \\
\hline
\end{tabular}

NOTE. Significance indicated in bold.

The effect size (Partial $\left.\eta^{2}\right)$ is as follows:

${ }^{a}$ Small (0.01-0.05).

${ }^{b}$ Medium (0.06-0.13).

${ }^{c}$ Large $(\geq 0.14)$.

Supplementary Table 3. Linear Regression Analysis, IBS Symptom Severity (z Score), $R^{2}=0.10$

\begin{tabular}{lcccc}
\hline & Estimate (B) & SE & $\beta$ & $P$ \\
\hline Constant & -0.495 & 0.090 & & .000 \\
Psychologic distress & $\mathbf{0 . 5 1 2}$ & $\mathbf{0 . 0 9 8}$ & $\mathbf{0 . 2 5 0}$ & $\mathbf{. 0 0 0}$ \\
Hypersensitivity & $\mathbf{0 . 3 2 5}$ & $\mathbf{0 . 0 9 8}$ & $\mathbf{0 . 1 6 0}$ & $\mathbf{. 0 0 0}$ \\
Abnormal transit & -0.03 & 0.112 & -0.014 & .773
\end{tabular}

NOTE. Factors independently associated with the outcome variables indicated in bold.
Supplementary Table 4.Linear Regression Analysis, Overall Somatic Symptom Severity (z Score), $R^{2}=0.17$

\begin{tabular}{lrrrr}
\hline & Estimate (B) & SE & $\beta$ & $P$ \\
\hline Constant & -0.549 & 0.086 & & .000 \\
Psychologic distress & $\mathbf{0 . 6 9 9}$ & $\mathbf{0 . 0 9 4}$ & $\mathbf{0 . 3 4 8}$ & $\mathbf{. 0 0 0}$ \\
Hypersensitivity & $\mathbf{0 . 3 6 1}$ & $\mathbf{0 . 0 9 3}$ & $\mathbf{0 . 1 8 1}$ & $\mathbf{. 0 0 0}$ \\
Abnormal transit & -0.040 & 0.107 & -0.017 & .709
\end{tabular}

NOTE. Factors independently associated with the outcome variables indicated in bold. 